\title{
Clinical criteria for the assessment of disease activity in Graves' ophthalmopathy: a novel approach
}

\author{
M Pн MOURITS,' L KOORNNEEF,' W M WIERSINGA,' M F PRUMMEL,' \\ A BERGHOUT, 2 AND $R \vee D$ GAAG $^{3}$ \\ From the 'Orbital Centre and the 'Department of Endocrinology of the University of Amsterdam, and the \\ ${ }^{3}$ Department of Immunology of the Netherlands Ophthalmic Research Institute, The Netherlands
}

\begin{abstract}
SUMmaRY Patients with serious inflammatory Graves' ophthalmopathy should be treated with anti-inflammatory drugs or radiotherapy to prevent complications like fibrosis, while those with non-inflammatory ophthalmopathy may be treated by surgery immediately. It is often difficult, however, to distinguish inflammatory from non-inflammatory Graves' disease. We therefore present a simple clinical classification here to differentiate between these two conditions. This classification is based on the classical signs of inflammation - pain, redness, swelling, and impaired function. After two consecutive clinical examinations an 'activity score' can be determined, ranging from 0 to 10 points. In a retrospective study testing the efficacy of this classification we found that patients with an activity score of 3 or more at the beginning of therapy responded well to anti-inflammatory drugs, while those with a lower activity score mostly did not. Comparing the pretreatment activity score with the degree of enlargement of the extraocular muscles on the CT scan, we found a significant correlation between these two parameters: the higher the activity score, the more the enlargement of the muscles. We conclude that this classification facilitates the proper selection of patients for treatment.
\end{abstract}

Graves' ophthalmopathy appears to be an organspecific autoimmune disorder.' A still unidentified pathogenic process induces swelling, lymphocytic infiltration, and later fibrosis and contractures that restrict the nornial function of the extraocular muscles." Two stages in the development of the disease are generally distinguished: the stage of active inflammation in which the eyes are red and painful, and the quiescent stage in which the eyes are white and unchanging with a painless motility deficit. ${ }^{3}$

Treatment of serious Graves' ophthalmopathy may be either non-surgical or surgical. Of the nonsurgical treatments orally administered steroids or radiotherapy or a combination of both are usually favoured. No agreement on the results of these treatments can be found. Brain ${ }^{4}$ reported immediate beneficial effects on the inflammatory components of the ophthalmopathy after short high doses of corticosteroids. Day and Caroll ${ }^{5}$ noted dramatic improvement of optic neuropathy by this therapy.

Correspondence to $\mathrm{M} \mathrm{Ph}$ Mourits, MD, Orbita Centrum, Academisch Medisch Centrum, A-2 116, Meibergdreef 9, 1105 AZ Amsterdam, The Netherlands.
McCohaneyt stated that extraocular muscle dysfunction at the stage of fibrosis and proptosis does not generally respond well to corticosteroids. However, Wiersinga et al.' found a positive response in $66 \%$ of all patients treated with prednisone alone. Hurbli et $a l .{ }^{*}$ found better results with radiation therapy in patients having a short history of Graves' ophthalmopathy than in patients with long-term ocular changes. Apart from basic differences in the definitions of successful treatment among authors these unequal success rates can be explained by the heterogeneity of the patients - patients with inflammatory progressive Graves' ophthalmopathy and those with the noninflammatory stationary stage of the disease.

The indication for treatment has always been the severity of symptoms (according to the NOSPECS classification*) instead of the rate of progression of the disease. During the initial stage immunosuppres-

\footnotetext{
*An abridged classification of eye changes in Graves ophthalmopathy ${ }^{1 / 10}$ : class (0): no physical signs or symptoms: (1) only signs, no symptoms (signs limited to upper lid retraction, stare, and lid lag); (2) soft-tissue involvement (symptoms and signs): (3) proptosis: (4) extraocular muscle movement: (5) corneal involvement: (6) sight
} 
sive treatment or radiotherapy is logical and inhibits the inflammatory process. Once fibrosis has taken place, this kind of treatment is useless. Surgery can deal with the residual sequelae successfully. It is therefore imperative to know whether a patient has active or quiescent Graves' ophthalmopathy.

To match appropriate therapy to the patients we propose a clinical classification to discriminate easily between the active and quiescent stages of the disease. This system is based on the well known signs of acute inflammation: pain (Latin: dolor), redness (rubor), swelling (tumor), and impaired function (functio laesa), defined by Celsus and Galen centuries ago.

\section{Clinical classification for disease activity}

Patients with Graves' ophthalmopathy complain of pain, a burning sensation, excessive lacrimation, photophobia, swelling of the eyelids, proptosis, double vision, and visual impairment. These symptoms and signs are related to the classical signs of inflammation (Table 1).

In Graves' ophthalmopathy several kinds of pain can be distinguished. One kind of pain arises from the stretching of the inflamed muscle(s), especially on attempted upgaze." Another sort of pain is caused by the rise in intraorbital pressure, when the orbital tissues expand through fluid accumulation and cellular infiltration. This is felt as a painful, oppressive feeling on or behind the globes. When asked, most patients can discriminate well between these two kinds of pain. The 'stretching pain' cannot be provoked by digital pressing on the eyeball, as would be expected if it were a manifestation of the raised intraorbital pressure. Both kinds of pain are the first

Table 1 Proposed classification system to assess disease activity in Graves' ophthalmopathy

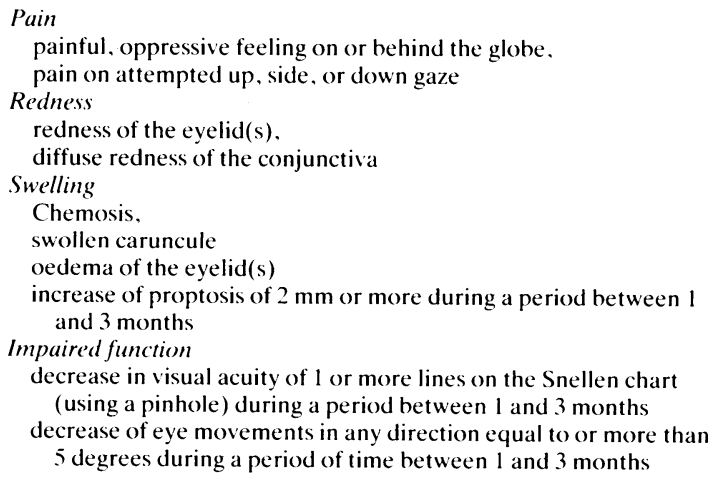

For each of the signs present one point is given. The sum of these points defines the activity score.

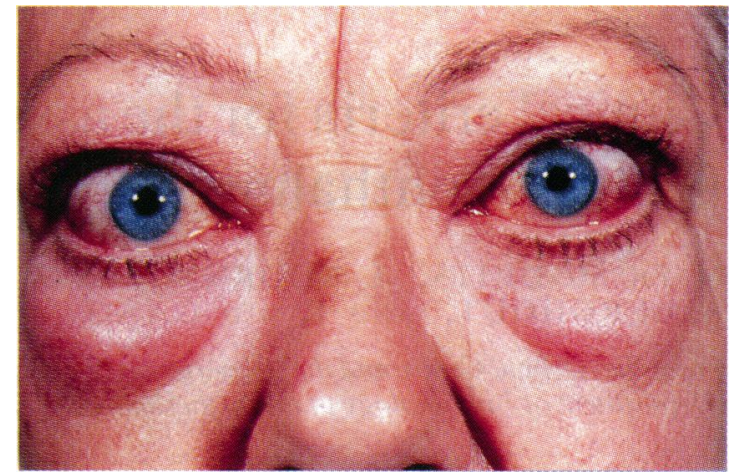

Fig. 1 Patient with inflammatory Graves' ophthalmopathy, showing redness of the eyelids and diffuse redness of the conjunctiva. Oedema of the eyelids is also clearly visible.

symptoms to disappear after anti-inflammatory treatment. We therefore consider these kinds of pain to be directly related to the inflammation in the orbit and thus useful in assessing inflammatory activity. They differ from pain caused by corneal defects, pain due to a spasm of the orbicularis muscle secondary to photophobia, or pain in the context of asthenopia because of a changed refraction. We consider these last symptoms to be epiphenomena and therefore not useful in assessing the disease activity.

Redness as an expression of inflammation is caused by vasodilatation. In Graves' ophthalmopathy this is seen as redness of the eyelids and over the conjunctiva (Fig. 1). These signs must be differentiated from the redness accompanying exposure keratitis and the grossly dilated vessels over the insertions of the extraocular muscles. The latter condition is seen both in active and in quiescent Graves' ophthalmopathy.

Swelling in Graves' ophthalmopathy is seen as chemosis (oedema of the conjunctiva) and a swollen caruncule (Fig. 2). Both are signs of activity. Swollen

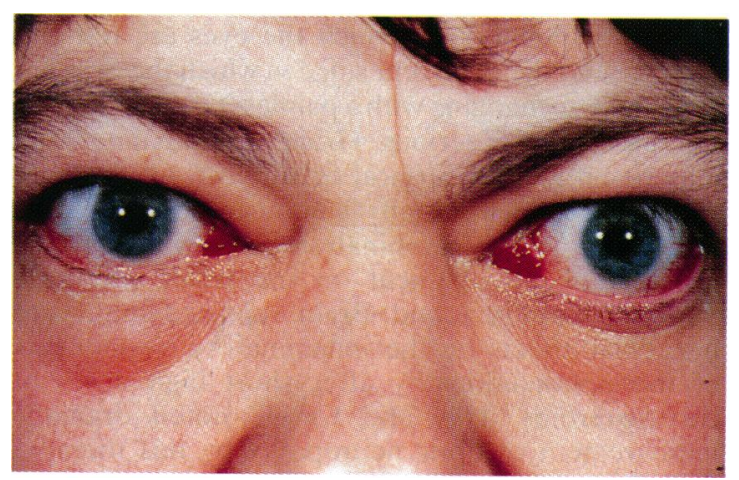

Fig. 2 Patient with inflammatory Graves'ophthalmopathy, showing grossly enlarged caruncles and oedema of the eyelids. 


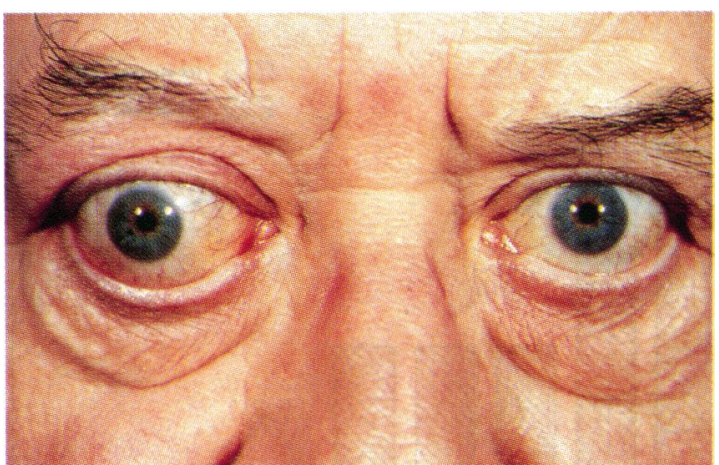

Fig. 3 Patient with 'burnt out' Graves' ophthalmopathy. The swelling of the eyelids is not caused by oedema.

eyelids can be caused by oedema, fat prolapse through the orbital septum, or fibrotic degeneration (Fig. 3). Here only eyelid oedema should be considered a sign of inflammation. To differentiate oedema from other causes of eyelid swelling palpation and illumination of the eyelids with a torch are mandatory.

In Graves' ophthalmopathy inflammation causes fluid accumulation and cellular infiltration in the orbit, later to be followed by fibrotic tissue. Proptosis and swelling of the eyelids are the result of the entire process. Therefore proptosis and eyelid swelling are not in themselves signs of acute inflammation. However, increasing proptosis in this context is a proof of it. A test-retest error of $2 \mathrm{~mm}$ is usually cited as the limit of exophthalmometer accuracy." "I We therefore consider an increase in proptosis of 2 or more millimetres to be a positive sign of disease activity.

Visual impairment in Graves' ophthalmopathy, resulting from dysfunction of the optic nerve, is caused by raised intraorbital pressure due to the inflammatory process. ${ }^{12}$ Therefore a decrease of visual acuity as a result of optic compression is a sign of disease activity. However, a decrease of visual acuity due to corneal defects cannot be considered an early sign of active Graves' disease. Corneal defects are most probably caused by increased width of the palpebral fissure, which accelerates tear film evaporation, thus increasing tear film osmolarity, with resulting ocular damage. ${ }^{13}$ That is why we consider corneal involvement to be an epiphenomenon. A decrease in eye movements implies that the extraocular muscle function is progressively impaired. It can therefore be regarded as a sign of disease activity.

To detect an increase in proptosis or a decrease in eye movements or visual acuity over a span of time at least two different consecutive examinations are necessary. In view of the natural history of Graves' ophthalmopathy we consider that the interval between these examinations should be at least one and not more than three months.

\section{Pilot study to test efficacy of classification}

The patients' records from an ongoing prospective, randomised, clinical trial to compare the efficacy, safety, and tolerability of cyclosporin and oral prednisone in severe Graves' ophthalmopathy ${ }^{14}$ were used for this purpose. In this study 26 consecutive patients with serious Graves' ophthalmopathy, defined as falling into NOSPECS categories $2 \mathrm{c}, 3 \mathrm{~b}$, $3 c, 4 b, 4 c, 5 a, 5 b, 5 c$, or $6 a$, were treated with oral prednisone or cyclosporin for a period of 12 weeks. These patients were euthyroid for more than two months when they started the trial. They had had no previous eye treatment other than local measures. Eighteen were female and eight male. Their ages ranged from 23 to 70 years.

The treatment consisted of $60 \mathrm{mg}$ prednisone/day during the first two weeks, $40 \mathrm{mg}$ during the next two weeks, $30 \mathrm{mg}$ during the next four weeks, and $20 \mathrm{mg}$ during the last four weeks, then tapering off, or cyclosporin $7.5 \mathrm{mg} / \mathrm{kg}$ bodyweight/day for 12 weeks. When the therapeutic outcome after 12 weeks was considered unsuccessful, the patients were treated for another 12 weeks with a combination of both oral prednisone $(20 \mathrm{mg} /$ day) and cyclosporin (in the same dosage as mentioned above). After 24 weeks the final outcome was evaluated. The success or failure of treatment was defined as shown in Table 2. Patients were examined before and at the beginning of therapy and after 1, 3, 4, and 6 months. Apart from classifying each patient in the NOSPECS system in each category, the visual acuity, proptosis, and eye movements were measured and eyelid oedema and chemosis recorded. At each visit photographs of the face were taken and the patients were asked about their complaints. Coronal CT scans of the orbits were carried out at the beginning of treatment.

The definitions of pain, as given in our scoring classification, could not be applied, as we were using

Table 2 Definitions of success and failure of treatment in Graves'ophthalmopathy

\footnotetext{
Success

A decrease (compared to baseline) in NOSPECS class or, if this did not occur, a decrease in grade within a class

Failure

An increase (compared to baseline) in NOSPECS class or. if this did not occur in grade within a class, or no change in NOSPECS class or grade
}

If both decreases and increases occurred in different classes, the change in the highest class was evaluated for therapeutic outcome. 
Fig. 4 Relationship between activity score and number of patients treated for serious Graves" ophthalmopathy.

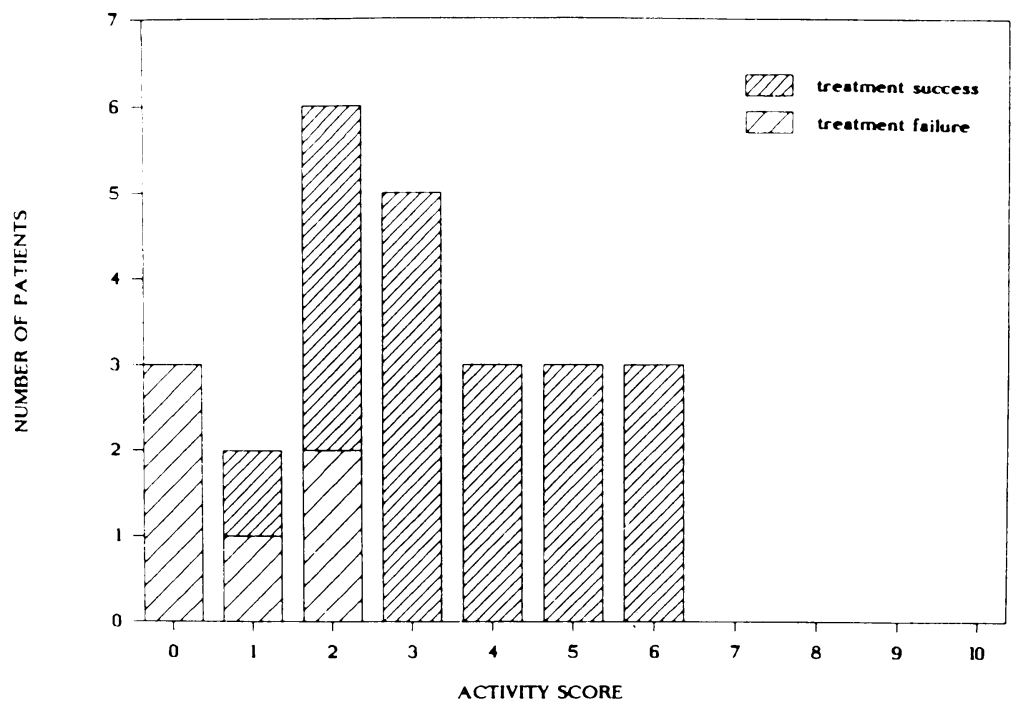

retrospective data, in which this item had not been planned for. However, all other signs of clinical activity could be assessed from the data.

The results of this study are shown in Fig. 4 and Table 3. After 24 weeks of therapy 19 out of 26 patients with serious Graves' ophthalmopathy had improved according to our predefined parameters and described standards. One patient (No. 24) underwent orbital decompression before the final evaluation of medical treatment was done, because of increasing visual impairment due to optic neuropathy (and was therefore not incorporated in Fig. 4). Three patients showed a worsening of their ophthalmopathy during treatment. They had an activity score of 1 or 2 points. The condition of the remaining three patients, all of them with an activity score of zero, did not change.

The degree of extraocular muscle enlargement on the orbital coronal CT scan was assessed in a rating system of 5 points ( 0 : no enlarged muscles; 5 : grossly enlarged muscles) by an ophthalmologist who was not informed about the activity score. By linear correlation and regression analysis a significant correlation was found between the extraocular muscle size and our activity score $(r=0.52$, volume $=$ $1 \cdot 9+0.31 \times$ activity score, $\mathrm{p}=0 \cdot 0007)$. The relationships between muscle size and the activity score will be studied in more detail in future prospective studies.

Fig. 4 suggests that the clinical activity score is

Table 3 Sex, age, relative incidence of disease activity signs, activity score, therapy, and results of therapy in 26 patients treated for serious Graves' ophthalmopathy

\begin{tabular}{|c|c|c|c|c|c|c|c|c|c|c|c|c|c|c|c|c|c|c|c|c|c|c|c|c|c|c|}
\hline Patient number & 1 & 2 & 3 & 4 & 5 & 6 & 7 & 8 & 9 & 10 & 11 & 12 & 1.3 & 14 & 15 & 16 & 17 & 18 & 19 & 20 & 21 & 22 & 23 & $24^{*}$ & 25 & 26 \\
\hline Sex & $\mathrm{F}$ & $\mathrm{F}$ & $\mathrm{F}$ & $\mathrm{M}$ & $\mathrm{F}$ & $\mathrm{F}$ & $\mathrm{F}$ & $M$ & $\mathrm{~F}$ & $\mathrm{~F}$ & $\mathrm{~F}$ & M & $\mathrm{F}$ & $\mathrm{F}$ & $\mathrm{M}$ & $\mathrm{F}$ & $\mathrm{F}$ & $\mathrm{F}$ & $\mathrm{M}$ & $\mathrm{F}$ & $\mathrm{M}$ & $\mathrm{M}$ & $\mathrm{F}$ & $\mathrm{F}$ & $\mathrm{F}$ & $M$ \\
\hline Age & 67 & 55 & 39 & 68 & 57 & 54 & 52 & 70 & 54 & 54 & 44 & 40 & 23 & 52 & 62 & 58 & 46 & 36 & 47 & 37 & 54 & 60 & 44 & 43 & 40 & 64 \\
\hline Pain & + & + & + & + & + & + & + & + & & & & & + & & & + & + & & + & & & & + & + & + & + \\
\hline Redness of eyelids & + & & + & + & + & & & + & + & + & + & & + & & + & + & + & & + & + & + & + & & + & & + \\
\hline Redness of conjunctiva & & & + & + & & & & + & & + & & & & & + & & + & & + & + & + & + & & + & & \\
\hline Chemosis & + & & & + & & & & & & & & & & & + & & + & & & + & + & + & & + & + & \\
\hline Swollen caruncula & & & & & & & & & & & & & & & & & + & & & + & & + & & + & & \\
\hline Eyelid oedema & & & + & + & + & & & + & & + & + & & + & & + & & + & & + & + & + & + & + & + & + & \\
\hline Increase proptosis & & & + & & & & & & & & & & & & & & & & & & & & & & & \\
\hline Decrease in VA & & + & + & + & & & + & + & & & & & & & & & & & & & & & & + & & \\
\hline Decrease in motility & & & & & & & & & & & & & & & & & & & & & & & & + & & \\
\hline Activity score & 3 & 2 & 6 & 6 & 3 & 1 & 2 & 5 & 1 & 3 & 2 & () & 3 & () & 4 & 2 & 6 & () & 4 & 5 & 4 & 5 & 2 & 8 & 3 & 2 \\
\hline Therapy & $\mathrm{D}$ & $\mathrm{D}$ & $\mathrm{C}$ & $A$ & C & $\mathrm{A}$ & $\mathrm{B}$ & $\mathrm{D}$ & D & C & A & D & A & $\mathrm{C}$ & I) & $\mathrm{D}$ & $\mathrm{A}$ & D & I) & A & I) & A & D & $\mathrm{C}$ & $\mathrm{B}$ & B \\
\hline Results of therapy & $\mathrm{S}$ & $\mathrm{S}$ & $\mathrm{S}$ & $\mathrm{S}$ & $\mathrm{S}$ & $\mathrm{S}$ & $\mathrm{S}$ & $\mathrm{S}$ & $\mathrm{F}$ & $\mathrm{S}$ & $\mathrm{S}$ & $\mathrm{F}$ & $\mathrm{S}$ & $\mathrm{F}$ & $\mathrm{S}$ & $\mathrm{F}$ & $\mathrm{S}$ & $\mathrm{F}$ & $\mathrm{S}$ & $\mathrm{S}$ & $\mathrm{S}$ & $\mathrm{S}$ & $\mathrm{F}$ & & $\mathrm{S}$ & $\mathrm{S}$ \\
\hline
\end{tabular}

*Patient No. 24 underwent orbital decompression before the final evaluation of medical treatment was made.

$\mathrm{F}=$ female $\mathrm{M}=$ male.

$\mathrm{A}=$ prednisone. $\mathrm{B}=$ cyclosporin. $\mathrm{C}=$ prednisone first, then cyclosporin added. $\mathrm{D}=$ cyclosporin first, then prednisone added.

$\mathrm{S}=$ treatment success. $\mathrm{F}=$ treatment failure. 
helpful in predicting the therapeutic outcome of immunosuppressive treatment in Graves' ophthalmopathy. In patients with an activity score of zero no change of the ocular condition could be demonstrated. Patients with a pretreatment activity score of less than 2 points showed no improvement, whereas patients with 3 or more points did improve on immunosuppressive treatment. In patients with an activity score of 2 points the result of this treatment could not be predicted.

\section{Discussion}

We propose a classification system for disease activity of Graves' ophthalmopathy which is easily manageable and entirely clinical, making it applicable without the use of special instruments. For this reason, the item 'heat' (Latin: calor), also one of the classical signs of acute inflammation, has been omitted, as the examiner would have difficulty diagnosing it without sophisticated instruments.

In the past patients have been treated with steroids and radiotherapy because of serious but not necessarily active Graves' ophthalmopathy. Our study suggests a positive correlation between our clinical activity score and a final favourable therapeutic outcome. This implies that patients with an activity score of 3 or more can expect beneficial effects from medical treatment. Those with a zero activity score should be spared from radiotherapy or steroids, of which the substantial side effects must not be forgotten. ${ }^{1+}$ They should be treated surgically. It is generally accepted that a period of at least six months has to elapse before surgical procedures can be applied, provided that no change in the NOSPECS classification has been observed. With our classification system this period ınay be considerably reduced. After two clinical examinations a few months apart disease activity can be assessed.

Support for the need of the above mentioned classification system can be derived from the literature. In 1969 Werner, ${ }^{15}$ as chairman of the American Thyroid Association ad hoc committee on eye disease, introduced the NOSPECS classification system for Graves' ophthalmopathy to summarise the most important symptoms once the diagnosis had been made. This system was modified in 1977. ${ }^{16}$ Although Werner's classification has been demonstrated to be of value for studying series of patients with comparable Graves' ophthalmopathy and for defining 'success and failure' in the treatment of the disease, it does not provide a means of distinguishing inflammatory progressive from non-inflammatory stationary Graves' ophthalmopathy. It is therefore of no help in deciding how and when to treat a patient. This is substantially illustrated by Figs. 1 and 3. Both patients had the same NOSPECS classification (namely, 2/2, 3/1, 4/2, 5/0, 6/0). The first patient (Fig. 1) clearly had active Graves' disease, the second had not.

Van Dijk, ${ }^{3}$ questioning the fact that the modified NOSPECS classification system no longer allowed disease activity to be specified, has proposed a second modification of the original classification, including a restoration of the listing of soft tissue signs. For class 2 he introduced the mnemonic RELIEF, trying to include designators of activity of the orbital process in the NOSPECS classification system. Although we agree that some of his designators clearly describe activity (for example, oedema of the caruncule, conjunctiva, and eyelid, and redness of the conjunctiva), other important symptoms and signs are omitted (for example, pain, redness of the eyelid, increase in proptosis, and impaired function), while finally two signs of questionable usefulness are mentioned (namely, resistance to retrodisplacement of the eye and lacrimal gland enlargement). We do not contest that these signs actually may occur in Graves' ophthalmopathy, but why should they indicate disease activity? Besides, according to Frueh et al. ${ }^{17}$ digital estimation of the resistance to retropulsion does not reliably suggest the measured forces. We believe that only eyelid swelling caused by oedema can be considered as a sign of activity, while fullness caused by a fat prolapse or infiltration cannot.

Kahaly et al. ${ }^{18}$ determined an activity score from the patient's history, clinical findings, and results of laboratory and imaging techniques. In this system signs and epiphenomena, such as eyelid closure and photosensitivity, are confused, while unchanging double vision and proptosis are included as signs of activity.

Sergott et al.," attempting to devise a disease activity index for Graves' ophthalmopathy to correlate with immunological parameters, found five clinical signs the best indicators of active, progressive ophthalmopathy. We have objections against two of these indicators. The sign of resistance to retrodisplacement has been discussed above. From our clinical experience we conclude that dilated blood vessels over the insertions of the horizontal extraocular muscles are a sign of both progressive and quiescent Graves' ophthalmopathy.

In our classification of disease activity we did not mention loss of colour vision because we consider that testing patients with Ishihara pseudoisochromatic colour plates gives less reproducible results and does not provide more information than already gathered by testing the visual acuity.

Although we are aware of the limitations of our pilot study, the results encouraged us to start a 
prospective study to show that our classification system is easily managed and increases the success rate of anti-inflammatory treatment in active Graves' ophthalmopathy.

We thank Mr P Y Moriarty, ophthalmic surgeon, for carefully reading and commenting on the manuscript.

\section{References}

1 Jacobson DH, Gorman CA. Endocrine ophthalmopathy: current ideas concerning etiology, pathogenesis and treatment. Endocr Rev 1984; 5: 200-20.

2 Gorman CA. Ophthalmopathy of Graves' disease. (Editorial.) N Engl J Med 1983; 308: 453-5.

3 van Dijk HJL. Orbital Graves' disease. A modification of the 'NOSPECS'-classification. Ophthalmology 1981; 88: 479-84.

4 Brian R. Cortisone in exophthalmos: report on a therapeutic trial of cortisone and corticotrophin (ACTH)) in exophthalmos and exophthalmic ophthalmoplegia by a panel appointed by the Medical Research Council. Lancet 1955; i: 6-9.

5 Day RM, Caroll FD. Corticosteroids in the treatment of optic nerve involvement associated with thyroid dysfunction. Trans Am Ophthalmol Soc 1967: 65: 41-51.

6 McCohany WH. The eye and orbit in thyroid disease. New York: Raven Press, 1984: 317-23.

7 Wiersinga WM, Smit T, Schuster-Uitterhoeve AJL, van der Gaag R, Koornneef $L$. Therapeutic outcome of prednisone and of orbital irradiation in patients with severe Graves' ophthalmopathy. Ophthalmologica 1988: 197: 75-84.

8 Hurbli T, Char DH, Harris J, Weaver R, Greenspan F, Sheline G. Radiation therapy for thyroid eye disease. Am J Ophthalmol 1985; 99: 633-7.
9 Sergott RC, Felberg NT, Savino PJ, Blizzard JJ, Schatz NJ. Graves' ophthalmopathy-immunologic parameters related to corticosteroid therapy. Invest Ophthalmol Vis Sci 1981: 20: 17383.

10 DeSanto LW. Transantral orbital decompression. In: Gormann CA. Waller RR, Dyer JA, eds. The eye and orbit in thyroid disease. New York: Raven Press, 1984: 236.

11 Char DH. Eye signs and diagnosis of thyroid ophthalmopathy. In: Gardner J, Vaughn V. Powell L, eds. Thyroid eye disease. Baltimore: Williams and Wilkins, 1985: 37.

12 Koornneef L. Eyelid and orbital fascial attachments and their clinical significance. Eye 1988: 2: 130-4.

13 Gilbard JP, Linsy Farrir R. Ocular surface drying and tear film osmolarity in thyroid eye disease. Acta Ophthalmol $(K h h) 1983$; 61: 108-16.

14 Prummel MF, Mourits MPh, Wiersinga WM, et al.. Cyclosporine versus prednisone in the treatment of severe Graves' ophthalmopathy. Preliminary results. (Abstr.) In: University of Texas Health Science Center, ed. The second international congress on cyclosporine. Washington DC, 1987: 92.

15 Werner SC. Classification of the eye changes of Graves' disease. J Clin Endocrinol Metab 1969: 29: 782-4.

16 Werner SC. Modification of the classification of the eye changes of Graves' disease. Am J Ophthalmol 1977; 83: 725-7.

17 Frueh BR, Musch DC, Grill R, Garber F. Hamby S. Orbital compliance in Graves' eye disease. Ophthalmology 1985: 92: $6.57-6.5$.

18 Kahaly G, Schrezenmeir J. Krause U, et al. Cyclosporine and prednisone .v. prednisone in treatment of Graves' ophthalmopathy, a controlled randomized and prospective study. Eur $J$ of Clin Invest 1986: 16: 415-22.

Accepted for publication 24 November 1988. 